\title{
Evaluation of a Botanical Extract that Mimics the Respiratory Cues of Cigarette Smoke
}

\author{
Jed E. Rose, Perry N. Willette, Tanaia H. Loeback, and David R. Botts \\ Rose Research Center, LLC, Raleigh, North Carolina
}

\begin{abstract}
ntroduction: Cigarette addiction results from both pharmacological effects of nicotine and the rewarding effects of associated cues, including respiratory tract sensations.

Aims: This study sought to evaluate the initial acceptability of a non-nicotine botanical formulation that provided similar respiratory tract cues.

Methods: Two active test products and matching placebos were evaluated. One test product, an e-cigarette-like device, delivered a visible aerosol upon puffing; the other test product delivered an invisible vapour at ambient temperature. Test products delivered a botanical extract with flavourings and vehicle; the placebos delivered flavourings and vehicle only. Sixteen participants had 3-h ad libitum access to each test product and associated placebos, and were deprived of combustible cigarettes for $1 \mathrm{~h}$ before and throughout the 3-h evaluation period. Subjects rated the satisfaction (primary outcome) and other sensory qualities of the products. Safety evaluations included pulmonary function testing and monitoring vital signs.

Results: Satisfaction ratings (seven-point scale) were significantly greater for the active e-cigarette-like condition; $M=3.18, S D=1.04$ versus $M=2.69, S D=1.22$. Safety evaluations showed no clinically significant changes.

Conclusions: The results support the potential acceptability of a non-nicotine cigarette substitute in providing satisfaction to smokers. This approach merits further evaluation for safety and acceptability in tobacco harm reduction and cessation.
\end{abstract}

\section{Introduction}

Smoking remains one of the leading causes of preventable morbidity and mortality throughout the world (Jamal, 2016). The World Health Organization (WHO) notes that tobacco use causes nearly six million deaths per year, with an estimated eight million deaths a year by 2030 , should current trends continue (WHO (2015)). The Centers for Disease Control and Prevention has stated that smoking is the leading cause of preventable death in the United States (Carter, Freedman, \& Jacobs, 2015), where there are an estimated 540,000 premature deaths a year due to cigarette smoking, and the economic cost of smoking is estimated to exceed $\$ 300$ billion a year (Xu, Bishop, Kennedy, Simpson, \& Pechacek, 2015). In addition to providing smokers with effective smoking cessation treatment options, new approaches are needed to substitute for the rewarding effects of smoking using less harmful alternatives.

Multiple studies have shown that habitual cigarette smoking is sustained via several factors, including the pharmacological effects of nicotine, as well as the behavioural and sensory cues associated with the act of smoking (Naqvi \& Bechara, 2005; Rose \& Behm, 2004). The limited success of current Food and Drug Administration (FDA)-approved smoking cessation treatments, which yield long-term success rates generally under $30 \%$ (Tobacco Use and Dependence Guideline Panel, 2008a; 2008b), may be due to the failure to provide smokers with satisfaction derived from smoking-related sensory cues. Among these cues are respiratory tract sensations, often referred to as 'impact' or 'throat hit' (Etter, 2016). The neurophysiological substrate mediating this chemosensory input from cigarette smoke is complex; major routes include sensory pathways involving the vagus nerve (cranial nerve $\mathrm{X}$ ), the trigeminal nerve (cranial nerve $\mathrm{V}$ ) and the glossopharyngeal nerve (cranial nerve IX) (Sentiens, LLC, 2012a). Local blockade of smoking-related respiratory tract sensations, using the local anesthetic lidocaine, or the peripherally-acting nicotinic receptor antagonist 
trimethaphan, acted to reduce smoking satisfaction and attenuate the ability of cigarette smoking to alleviate craving (Rose, Westman, Behm, Johnson, \& Goldberg, 1999; Rose, Zinser, Tashkin, Newcomb, \& Ertle, 1984).

Smoking-related respiratory tract sensations can be simulated without inhalation of nicotine or toxic products of burning tobacco. For example, in previous studies, inhalation of an aerosol containing citric acid or ascorbic acid reduced craving for cigarettes (Behm, Schur, Levin, Tashkin, \& Rose, 1993; Levin et al., 1993); a clinical trial using a cigarette-sized dry powder citric acid delivery system subsequently showed efficacy in smoking cessation treatment relative to a lactose placebo, when combined with nicotine replacement therapy (NRT) (Westman, Behm, \& Rose, 1995). In other studies, inhalation of minute quantities of capsaicin, or vapour from black pepper essential oil, replicated some of the respiratory tract sensations associated with cigarette smoking and provided partial satisfaction of the desire for a cigarette (Behm \& Rose, 1994; Rose \& Behm, 1994).

A botanical extract containing chemosensory stimulating constituents has been developed commercially ('BotanicBoost', Novus brand products, produced by Sentiens, LLC, Charlotte, NC), which, upon inhalation, mimics the respiratory tract chemosensory cues associated with inhalation of nicotine. The proprietary botanical extract includes (listed alphabetically) small amounts of Aframomum meleguate extract, allspice extract, cedar absolute extract, diluent (glycerin and propylene glycol), ethanol, extract from Eucalyptus, eugenol oil, galangal, mustard seed extract, rosemary extract, Szechuan pepper extract and thyme leaves (US8646461 B2; von Borstel, Tan, Siverling, \& Timokhina, 2014). The microgram-level quantities of these constituents, all of which have been classified as 'generally regarded as safe' (GRAS), represent levels significantly lower than found in a typical meal made with the constituent spices. Also included in the extract was flavouring (without nicotine) derived from tobacco. Since this product has been marketed prior to August 8, 2016, pre-market review was not required according to the relevant FDA guidance for industry (FDA (2016)). Additional safety information is available from the manufacturer via White Papers (Sentiens, LLC, 2012b; Sentiens, LLC, 2012c).

The purpose of this study was to evaluate the ability of two products containing this botanical extract-one delivering an aerosol in an e-cigarette-like device, and one delivering an invisible vapour from a porous cigarettesized rod-to provide satisfaction to smokers during a period of smoking abstinence. Secondary outcomes included strength and harshness ratings of each product. The main goal of the study was limited: to determine whether smoking-related sensations could be mimicked by the botanical extract evaluated, in order to produce an incremental change in subjective satisfaction. Nicotine was not the focus of this study, and hence a direct comparison with combustible cigarettes was not included. This study did not assess efficacy or effectiveness for smoking cessation. Pending the successful development of an adequate sensory substitute for cigarette smoking, this formulation could ultimately be combined with methods for delivering nicotine, with the goal of sustaining a level of overall reward and satisfaction that might successfully compete with cigarette smoking.

\section{Methods \\ Design}

A randomised, double-blind, placebo-controlled, counterbalanced $2 \times 2$ designed study compared active versus placebo e-cigarette-like products and active versus placebo invisible vapour products. The protocol was reviewed and approved by the Schulman Institutional Review Board (IRB) to ensure the study was conducted in accordance with Good Clinical Practice (GCP) based on guidelines from the current International Conference on Harmonisation ( $\mathrm{ICH}$ ), the basic principles of the Declaration of Helsinki, United States Code of Federal Regulations governing protection of human subjects (Title 21 CFR Part 50 and Title 21 CFR Part 56) and all applicable legal and regulatory requirements. External funding for this study was limited to supplying of study products by Sentiens, LLC, which has a sponsored research and commercialisation agreement with the study centre. The study recruited subjects from Raleigh and Charlotte, North Carolina, utilising a private database of over 10,000 cigarette smokers who have expressed interest in participating in research studies. The database was generated using IRB approved generic advertisements. Current cigarette smokers (men and women) between the ages of 19 and 65 years of age, with no restriction on race or ethnicity, were recruited for this study. Criteria for participation included smoking on average at least 10 commercially available combustible cigarettes per day, having an exhaled carbon monoxide level of at least 10 parts per million at the first visit and no indicated intention of quitting smoking within 60 days of study enrolment. Subjects were excluded if they had abnormal pulmonary function test (evidence of restrictive or obstructive lung disease), had difficulty providing blood or urine samples, had uncontrolled psychiatric disease by self-report or as noted on the Patient Health Questionnaire (PHQ-9), were pregnant or breastfeeding, had elevated scores on the Alcohol Use Disorder Identification Test (AUDIT) or reported chronic use of opioids or daily use of sleep aids. Additionally, subjects were excluded if they used illicit drugs (by self-report or as noted on an unexplained positive urine drug screen for amphetamines, methamphetamines, barbiturates, benzodiazepines, cannabinoids, opiates, buprenorphine, methadone, oxycodone, phencyclidine, propoxyphene or tricyclic antidepressants), used NRT or used non-combustible tobacco products within 14 days of the initial screening visit. Each subject was evaluated by a physician and determined to be healthy and free of 
any significant medical issues. After obtaining informed consent, the study enrolled eligible adult male and female current cigarette smokers. Each subject could participate in evaluation of both the e-cigarette-like product and the invisible vapour delivery product, matched to the flavouring corresponding to their usual brand cigarette (tobacco flavour or menthol flavour). However, if a subject's participation ended before completing all four sessions, the data for active and placebo conditions for the product sessions they completed were used. As it happened, 15 of 16 subjects in the vapour product condition also participated in the e-cigarette-like product evaluation. One subject did not complete the e-cigarette-like product (menthol flavour) evaluation sessions, requiring enrolment of one additional subject. Research staff and subjects remained blinded to the specific product (active versus placebo) throughout the entire study.

Seventeen subjects were enrolled at sites in Raleigh and Charlotte, North Carolina in order to obtain 16 complete sets of data for each test product (and matching placebo). For each test product, eight subjects were assigned to receive the 'menthol' flavor and eight subjects were assigned to receive the 'tobacco' flavour, corresponding to the flavour of their usual brand of cigarette. Subjects were asked to attend four laboratory sessions, each conducted after $1 \mathrm{~h}$ of supervised abstinence from any tobacco/nicotine containing products. During each 3-h laboratory session, subjects were not allowed to smoke but could use the assigned product ad libitum. The order of product use for each laboratory session was randomly assigned at enrolment using one of eight sequences of product exposure, which counterbalanced the order of active versus placebo products and the order of e-cigarette-like versus invisible vapour products.

Participants were administered sensory questionnaires every $30 \mathrm{~min}$ during the 3 -h ad libitum period. The questionnaire assessed ratings of satisfaction, liking, harshness, similarity to the usual brand of cigarettes and strength of sensations in different regions of the respiratory tract. The initial screening session lasted approximately $3 \mathrm{~h}$, and each laboratory session lasted approximately $5 \mathrm{~h}$ (Figure 1). All five visits (screening and four laboratory sessions) were completed within an 8-week period, with a minimum of $24 \mathrm{~h}$ between each session.

Lung function was analysed by obtaining pulmonary function testing, including Pulmonary Function Tests (PFTs, spirometry) at screening, and at the end of each 3-h ad libitum use period. Measurements taken, in accordance with 2005 guidelines of the American Thoracic Society (ATS)/European Respiratory Society (ERS) Joint Task Force on Standardisation of spirometry, were forced expiratory volume in one second (FEV1), forced vital capacity (FVC) and forced expiratory flow during the midportion of forced expiration (FEF25-75). Peak expiratory flow rate (PEFR) measurements were obtained as a measurement of acute responses, and were recorded at baseline and at $60-\mathrm{m}$ intervals during the $3-\mathrm{h}$ ad libi-

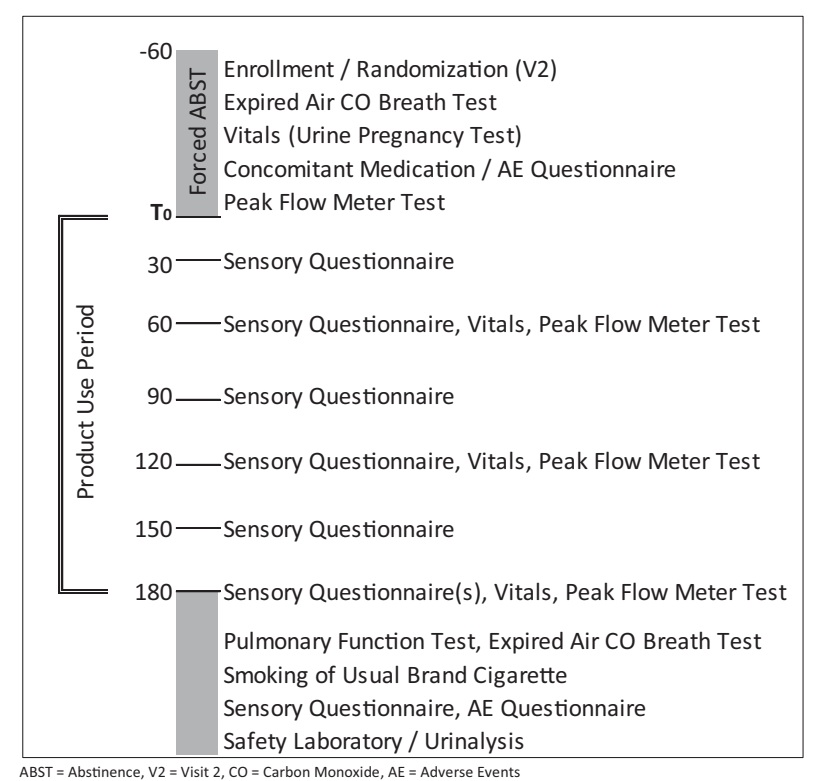

Figure 1

Laboratory session schedule of events. ABST = abstinence, V2 = visit 2, $\mathrm{CO}=$ carbon monoxide, $\mathrm{AE}=$ adverse events.

tum sessions. PEFR testing was performed in accordance with the National Institutes of Health (NIH)/National Heart, Lung, and Blood Institute (NHLBI) guidelines. Vital signs, including blood pressure, heart rate and respiratory rate were measured at the beginning of each session and at 60-min intervals until the end of each session.

\section{Test Products}

The active e-cigarette-like test product used a batteryoperated coil to heat a vehicle containing chemostimulating botanical extract, propylene glycol, tobacco flavouring extracted from tobacco and other flavourings. It was similar in shape and size to many e-cigarette products on the market, with the same length and circumference of a combustible cigarette and an actuating button that is pressed to generate the vapour for inhalation. The placebo ecigarette-like device was identical to the test product, and delivered all constituents except for the botanical extract.

The active invisible vapour delivery system consisted of a $100 \%$ paper rod, the same size and shape as a cigarette, with a porous plug infused with ethanol, flavouring and the botanical extract. It uses the same outer paper and tipping paper as cigarettes, with the inner paper infused with the botanical extract. Again, the placebo test product was identical except for the absence of the botanical extract. None of the test products contained nicotine. Both active test products were commercially available as of August 8, 2016, and hence were exempt from a pre-market authorisation requirement by FDA's Center for Tobacco Products (FDA (2017)). 


\section{Statistical Analyses}

As delineated a priori in the IRB-approved protocol, a single primary outcome was designated: the self-reported rating of satisfaction, using a scale ranging from 1 (not at all) to 7 (extremely). Ratings were compared between each active product and its respective placebo using paired t-tests. Given the a priori designation of a single outcome measure, no correction for multiple statistical comparisons was made; secondary outcome comparisons were viewed as purely exploratory. Also, in view of the unambiguous directional nature of the hypothesis (i.e., that the active formulation would increase, rather than decrease satisfaction ratings, based on several previous studies in which stimulation of respiratory tract sensations elicited satisfaction), one-tailed statistical tests were employed. Additional subjective ratings (e.g., liking, harshness, similarity to usual brand of cigarette and strength of sensation in different regions of the respiratory tract) were collected and tabulated for descriptive purposes, without conducting statistical comparisons. Safety was evaluated by assessment of vital sign measurements and pulmonary function testing (using spirometry and measuring peak expiratory flow rates).

Based on a predicted large effect size for the difference between active and placebo conditions (Cohen's $d=1.0$ ), a sample size of 16 for each within-subject comparison would yield a power of greater than $80 \%$ to detect the expected differences.

\section{Results}

Subjects in the e-cigarette-like device test product evaluation included seven males, nine females (nine whites, one Asian and six African Americans) had a mean age of 42.67 years $(S D=8.11)$ and smoked on average 16.06 cigarettes/day $(\mathrm{SD}=5.11)$ with an FTND (Fagerstrom Test for Nicotine Dependence) score of 5.31 (SD = 1.92). Subjects in the invisible vapour test product evaluation included six males, 10 females (nine whites, one Asian and six African Americans), had a mean age of 42.41 years $(\mathrm{SD}=8.24)$ and smoked on average 15.56 cigarettes/day $(\mathrm{SD}=5.09)$ with an FTND score of $5.31(\mathrm{SD}=1.92)$. One additional subject was enrolled in order to obtain eight complete data sets for the menthol smoking group (one subject failed to attend both e-cigarette-like device test product sessions).

Mean satisfaction ratings were significantly greater for the active e-cigarette-like device condition $(M=3.18$, $\mathrm{SD}=1.04)$ than for the placebo e-cigarette-like device $(M=2.69, \mathrm{SD}=1.22) ; t(15)=2.26, P=0.02$, one-tailed. Although a one-tailed test was specified a priori, and justified based on the previously mentioned statistical analyses, this test would have yielded a statistically significant $P=0.04$ had a two-tailed test been conducted. There was also a trend for the vapour condition to be rated higher than the placebo $(M=2.27, \mathrm{SD}=1.45$ versus $M=1.83$, $\mathrm{SD}=1.22$ ), with this comparison falling short of statisti- cal significance; $t(15)=1.68, P=0.06$ (one-tailed). Other subjective ratings are depicted in Table 1.

No serious adverse events were observed, and there were no clinically significant changes in vital signs or pulmonary functions associated with product use. Sitting heart rates for all subjects remained within normal ranges with the use of the active e-cigarette-like product, the ecigarette-like placebo, the invisible vapour product and the invisible vapour placebo product (see Table 2). Mean arterial pressures also remained stable, with no significant changes associated with the use of any of the products or placebos (Table 2).

Analysis of lung function showed no clinically significant effects on spirometry (FEV1/FVC or FEF25-75) for any subjects after using either of the products or their placebos (Table 2). PEFRs also showed no significant effects associated with the use of either of the products evaluated or their corresponding placebos (Table 2).

\section{Discussion}

The active chemosensory formulation delivered in an e-cigarette-like platform provided moderate levels of satisfaction to cigarette smokers, which were significantly higher than the placebo. Similarly, there was a trend for the active invisible vapour product to be rated higher in satisfaction than the corresponding placebo. To place the satisfaction ratings in perspective, in a study of 41 cigarette smokers using marketed nicotine-containing products for three days, Steinberg et al. reported mean satisfaction ratings of 5.0 for a popular brand of ecigarette and 2.6 for the FDA-approved nicotine inhaler (Steinberg et al., 2014). Thus, the current active ecigarette-like test product, despite the absence of nicotine, compared favourably with at least one marketed nicotinecontaining product. Although not formally compared, the e-cigarette-like product tended to be rated more satisfying than the invisible vapour product, which is not surprising given that the former delivered an aerosol that elicited respiratory tract sensations more similar to those of cigarette smoke, which is itself an aerosol. Further studies will be needed in order to evaluate whether this is a reliable difference between the two types of products.

It should be noted that the placebo products were themselves 'active' in the sense that they provided behavioural substitution components (handling, puffing) as well as flavour and (in the case of the e-cigarette-like test product) visual sensory cues that mimicked those of cigarette smoke. Thus, the overall impact of active product use compared to a no-device control condition would likely have been greater than the active-placebo difference noted here. Also, we are aware of no other published studies showing that a currently marketed product containing no nicotine elicits greater satisfaction than placebo. Given this dearth of acceptable non-nicotine alternatives for cigarettes, our findings, while preliminary, 


\begin{tabular}{lllll}
\hline $\begin{array}{l}\text { Table 1 } \\
\text { Results }\end{array}$ & \multicolumn{1}{l}{} \\
\hline Questions & e-Cigarette-Like Device & e-Cigarette-Like Placebo & Vapour & Vapour Placebo \\
\hline How satisfying were the puffs you took? & $3.18(1.04)$ & $2.69(1.22)$ & $2.27(1.45)$ & $1.83(1.22)$ \\
How much did you like the puffs you took? & $3.54(0.89)$ & $3.32(1.24)$ & $2.70(1.50)$ & $2.07(1.15)$ \\
How harsh were the puffs you took? & $2.41(1.14)$ & $1.88(1.16)$ & $1.93(1.13)$ & $1.73(1.23)$ \\
How similar to your own brand were the puffs? & $1.99(1.11)$ & $1.98(1.23)$ & $1.64(1.22)$ & $1.25(0.49)$ \\
Strength of puffs on tongue? & $2.38(1.21)$ & $2.10(1.15)$ & $2.24(1.06)$ & $1.91(1.06)$ \\
Strength of puffs in nose? & $1.79(1.20)$ & $1.68(1.20)$ & $1.73(1.03)$ & $1.51(0.98)$ \\
Strength of puffs in the back of the mouth \& throat? & $2.47(1.20)$ & $1.95(1.20)$ & $2.39(1.21)$ & $1.80(1.05)$ \\
Strength of puffs in windpipe? & $2.42(1.27)$ & $1.93(1.35)$ & $1.96(1.12)$ & $1.63(1.01)$ \\
Strength of the puffs in chest? & $1.97(1.33)$ & $1.78(1.36)$ & $1.63(1.10)$ & $1.42(0.81)$ \\
\hline
\end{tabular}

Values are reported as mean (standard deviation). Ratings are on a seven-point scale ranging from 1 (not at all) to 7 (extremely). This questionnaire was administered every 30 min during the product use periods.

\section{Table 2}

Vital signs and pulmonary functions

\begin{tabular}{|c|c|c|c|c|c|}
\hline Safety Data & Screening/Baseline & e-Cigarette-Like Device & e-Cigarette-Like Placebo & Vapour & Vapour Placebo \\
\hline \multicolumn{6}{|l|}{ Heart rate } \\
\hline bpm (SD) & $72.6(12.2)$ & $74.7(10.8)$ & $73.3(13.3)$ & $74.0(10.8)$ & $73.8(12.5)$ \\
\hline \multicolumn{6}{|l|}{ MAP } \\
\hline $\mathrm{mmHg}(\mathrm{SD})$ & $90.3(9.2)$ & $91.0(10.8)$ & $92.0(9.8)$ & $92.5(10.6)$ & $92.6(8.5)$ \\
\hline \multicolumn{6}{|l|}{ FEV1/FVC } \\
\hline$\%(S D)$ & $78.0(4.5)$ & $77.3(5.8)$ & $77.9(6.9)$ & $79.3(5.9)$ & $79.6(6.0)$ \\
\hline \multicolumn{6}{|l|}{ FEF $25-75$} \\
\hline L/S (SD) & $2.86(0.94)$ & $2.84(1.42)$ & $2.71(0.91)$ & $3.01(0.85)$ & $2.90(0.87)$ \\
\hline \multicolumn{6}{|l|}{ PEFR } \\
\hline $\mathrm{L} / \mathrm{s}(\mathrm{SD})$ & $8.03(1.98)$ & $7.90(1.94)$ & $7.75(1.83)$ & $8.02(1.85)$ & $7.68(1.90)$ \\
\hline
\end{tabular}

Values are reported as mean (standard deviation).

bpm: beats per minute. MAP: mean arterial pressure. mmHg: millimetres of mercury. SD: standard deviation. L/s: litres per second. FEV1/FVC: Forced expiratory volume in one second/forced vital capacity ratio at screening and end of each 3-h ad libitum session. FEF 25-75: Forced expiratory flow during the mid-portion of forced expiration at screening and end of each 3-h ad libitum session. PEFR: Peak expiratory flow rate at baseline, prior to any testing, and averaged over each 3 -h ad libitum session.

offer a novel and potentially important line of future investigation.

The safety evaluation showed no adverse effects of using the test products in an acute 3-h session. Lung function parameters, including FEV1/FVC, PEFR and FEF2575 showed no clinically significant changes from baseline. Further studies will be clearly needed to evaluate safety with longer-term use.

In conclusion, the study results suggest that a nonnicotine formulation that stimulates sensory nerve endings in the respiratory tract may be a fruitful approach to providing a degree of satisfaction to cigarette smokers. This approach merits further study as a practical approach to tobacco harm reduction by reducing consumption of combustible cigarettes. Depending on the context, the e-cigarette-like version or the invisible vapour version might provide more acceptability or conform to regulatory restrictions. In addition to evaluating the potential for smoking reduction, the technology merits further evaluation for acceptability and efficacy in smoking cessation treatment, alone or in combination with NRT.

\section{Acknowledgements}

The authors would like to thank Tammy Esarove, Derek Mercedes, LaKeshia Scott, Megan Vann, Rebecca Walters and Antionette White for their invaluable work in completing this study.

\section{Financial Support}

This work was supported by Rose Research Center, LLC. Study products were provided by Sentiens, LLC.

\section{Conflict of Interest}

Dr. Rose, Ms. Loeback and Mr. Botts have an equity interest in Rose Research Center, LLC, and Dr. Willette is an 
employee of Rose Research Center, LLC, which has sponsored research and commercialisation agreements with Sentiens, LLC.

\section{Ethical Standards}

The authors assert that all procedures contributing to this work comply with the ethical standards of the relevant national and institutional committees on human experimentation and with the Helsinki Declaration of 1975, as revised in 2008 .

\section{References}

Behm, F. M., \& Rose, J. E. (1994). Reducing craving for cigarettes while decreasing smoke intake using capsaicinenhanced low tar cigarettes. Experimental and Clinical Psychopharmacology, 2(2), 143-153.

Behm, F. M., Schur, C., Levin, E. D., Tashkin, D. P., \& Rose, J. E. (1993). Clinical evaluation of a citric acid inhaler for smoking cessation. Drug and Alcohol Dependence, 31(2), 131-138.

Carter, B. D., Freedman, N. D., \& Jacobs, E. J. (2015). Smoking and mortality-beyond established causes. The New England Journal of Medicine, 372(22), 2170.

Etter, J.-F. (2016). Throat hit in users of the electronic cigarette: An exploratory study. Psychology of Addictive Behaviors, $30(1), 93-100$.

FDA (2016). 81 FR 28974. Retrieved from https: //www.fda.gov/downloads/TobaccoProducts/Labeling/ RulesRegulationsGuidance/UCM524918.pdf

FDA (2017). Rules, regulations \& guidance. Retrieved June 23, 2017 from https://www.fda.gov/TobaccoProducts/Labeling/ RulesRegulationsGuidance/default.htm

Jamal, A. (2016). Current cigarette smoking among adultsUnited States, 2005-2015. Morbidity and Mortality Weekly Report, 65, 1205-1211.

Levin, E. D., Behm, F., Carnahan, E., LeClair, R., Shipley, R., \& Rose, J. E. (1993). Clinical trials using ascorbic acid aerosol to aid smoking cessation. Drug and Alcohol Dependence, 33(3), 211-223.

Naqvi, N. H., \& Bechara, A. (2005). The airway sensory impact of nicotine contributes to the conditioned reinforcing effects of individual puffs from cigarettes. Pharmacology, Biochemistry, and Behavior, 81(4), 821-829. doi: 10.1016/j.pbb.2005.06.005.

Rose, J., \& Behm, F. (2004). Effects of low nicotine content cigarettes on smoke intake. Nicotine \& Tobacco Research, 6(2), 309-319.

Rose, J. E., \& Behm, F. M. (1994). Inhalation of vapor from black pepper extract reduces smoking withdrawal symptoms. Drug and Alcohol Dependence, 34(3), 225-229.
Rose, J. E., Westman, E. C., Behm, F. M., Johnson, M. P., \& Goldberg, J. S. (1999). Blockade of smoking satisfaction using the peripheral nicotinic antagonist trimethaphan. Pharmacology, Biochemistry, and Behavior, 62(1), 165-172.

Rose, J. E., Zinser, M. C., Tashkin, D. P., Newcomb, R., \& Ertle, A. (1984). Subjective response to cigarette smoking following airway anesthetization. Addictive Behaviors, 9(2), 211215.

Sentiens, LLC (2012a). SENTIENS SCIENCE - Chemosensation. Retrieved February 7, 2017 from http://www.sentiens. us/chemosensation

Sentiens, LLC (2012b). SENTIENS SCIENCE - Guiding Principles and Safety. Retrieved February 2, 2018 from https: //www.sentiens.us/safety-gateway-theories

Sentiens, LLC (2012c). SENTIENS SCIENCE - Novus for Healthcare Professionals. Retrieved February 2, 2018 from https://www.sentiens.us/for-healthcare-professionals

Steinberg, M. B., Zimmermann, M. H., Delnevo, C. D., Lewis, M. J., Shukla, P., Coups, E. J. et al. (2014). E-cigarette versus nicotine inhaler: Comparing the perceptions and experiences of inhaled nicotine devices. Journal of General Internal Medicine, 29(11), 1444-1450. doi: 10.1007/s11606014-2889-7

Tobacco Use and Dependence Guideline Panel (2008a). Evidence and Recommendations. US Department of Health and Human Services, Washington, DC. Retrieved from https: //www.ncbi.nlm.nih.gov/books/NBK63943/

Tobacco Use and Dependence Guideline Panel (2008b, May). Table 6.26, Meta-analysis (2008): Effectiveness and abstinence rates for various medications and medication combinations compared to placebo at 6-months postquit ( $\mathrm{n}=83$ studies). US Department of Health and Human Services, Washington, DC. Retrieved June 21, 2017 from https://www.ncbi.nlm.nih.gov/books/NBK63958/table/ A29582/

von Borstel, R., Tan, D., Siverling, J., \& Timokhina, I. S. (2014). U.S. Patent US8646461 B2, Device and method for simulating chemosensation of smoking, February 11, 2014. Retrieved from http://www.google.com/patents/US8646461

Westman, E. C., Behm, F. M., \& Rose, J. E. (1995). Airway sensory replacement combined with nicotine replacement for smoking cessation. A randomized, placebo-controlled trial using a citric acid inhaler. Chest, 107(5), 1358-1364.

WHO (2015). Noncommunicable diseases. Retrieved February 7, 2017 from http://www.who.int/mediacentre/factsheets/ fs355/en/

Xu, X., Bishop, E. E., Kennedy, S. M., Simpson, S. A., \& Pechacek, T. F. (2015). Annual healthcare spending attributable to cigarette smoking: An update. American Journal of Preventive Medicine, 48(3), 326-333. doi: 10.1016/j.amepre.2014.10.012. 